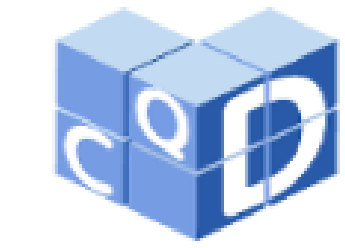

Revista Eletrônica

Paulista de Matemática

ISSN 2316-9664

Volume 21, dez. 2021

Walter Martin

Colégio Lúdico Paulista

waltermartin101@gmail.com

Maria Teresa Zampieri

Universidade Federal de São

Carlos

maria.zampieri@ufscar.br

\section{Um estudo sobre somas sequenciais como pro- postas didáticas investigativas para o ensino de Matemática}

A study on sequential sums as investigative didactic proposals for the teaching of Mathematics

\begin{abstract}
Resumo
O objetivo desse trabalho é discorrer sobre uma investigação matemática referente a Somas Numéricas Sequenciais, com vistas a tecer uma proposta didática investigativa, voltada para a Educação Básica, a ser analisada e implementada por licenciandos em Matemática, como parte das tarefas desenvolvidas nas disciplinas de Estágio Supervisionado de Matemática na Educação Básica, de cursos de Licenciatura em Matemática. $\mathrm{O}$ artigo apresenta um estudo qualitativo, caracterizado como documental, uma vez que faz uso de fontes primárias como: manuscrito com a investigação realizada, documentos curriculares oficiais nacionais e estaduais e textos teóricos. Por fim, almeja-se destacar os pormenores da investigação realizada, ressaltando possibilidades para sua problematização na formação inicial de professores de Matemática e com professores já em exercício, com vistas a adentrarem futuramente à Educação Básica.
\end{abstract}

Palavras-chave: investigação matemática. matemática escolar. educação matemática crítica.

\begin{abstract}
The objective of this paper is to discuss a mathematical investigation regarding Sequential Numerical Sums, with a view to making an investigative didactic proposal, focused on Basic Education, to be analyzed and implemented by undergraduate students in Mathematics, such as part of the tasks developed in the disciplines of Supervised Internship in Mathematics in Basic Education, which make up the curriculum of the Mathematics Degree courses. The paper presents a qualitative study, characterized as documentary, since it makes use of primary sources such as: manuscripts with the research carried out, official national and state curricular documents and theoretical texts. Finally, it aims to highlight an investigative process carried out with elementary mathematics, highlighting possibilities to be problematized in the initial education of mathematics teachers, with a view to entering into Basic Education in the future.
\end{abstract}

Keywords: mathematical research, school mathematics, critical mathematics education 


\section{Introdução}

Há décadas que a comunidade acadêmica vem debatendo alternativas para o ensino e a aprendizagem de Matemática, de modo que os estudantes se engajem mais nesse processo, desenvolvendo sua autonomia e tendo o professor como um orientador.

A importância dessa temática fica mais evidente se levarmos em consideração o momento atual em que estamos vivendo, que é o contexto da pandemia do Novo Coronavírus, uma vez que a rotina escolar mudou bruscamente, o que acabou demandando novos esforços por parte de alunos e professores. Já esse vírus, por sua vez, "pegou todo mundo de surpresa", conforme muitos indivíduos costumam opinar, seja em uma roda de conversa entre amigos, seja por meio das redes sociais, ou em quaisquer outros veículos de comunicação. Mas será que isso foi mesmo uma surpresa?

Para o pesquisador Ubiratan D'Ambrosio, e, para quem já o ouviu em algumas de suas provocativas palestras, certamente responderão que não. Nessas diferentes ocasiões, Ubiratan, ao dialogar com importantes referências na área de Matemática - como o matemático russo/francês Mikhail Leonidovjch Gromov, vencedor do prêmio Abel de 2009 - destacou diferentes problemas que a Terra poderia passar como consequência do crescimento insustentável da população, e, mencionou que a rápida disseminação de pandemias seria um deles.

Em sua palestra proferida no VII Congresso Ibero-americano de Educação Matemática, em Montevidéu no Uruguai, em 2013, o pesquisador reforçou essa preocupação: "Há um perigo evidente de extermínio da civilização devido a problemas meteorológicos e geoestruturais, mas também por conflitos gerados por seres humanos. Tal impasse nos desafia a construir novas direções para entender, explicar e agir no mundo real [...]" (D'AMBROSIO, 2014, p. 157). E então nos parece natural a seguinte indagação: mas como educadores e gestores, o que podemos fazer?

No nosso modo de ver, uma possibilidade seria pensar e incentivar o desenvolvimento de propostas didáticas investigativas, junto aos alunos, e que, de algum modo, despertassem a curiosidade desses estudantes, para que eles tivessem mais autonomia em sua aprendizagem, com a orientação do professor. Nessa direção, Skovsmose (2000) destaca a importância de que reflitamos sobre o desenvolvimento de uma educação matemática "que não torne opaca a introdução dos alunos ao pensamento matemático, mas que os leve a reconhecerem suas próprias capacidades matemáticas e a consciência da forma pela qual a matemática opera [...]" (SKOVSMOSE, 2000, p. 19).

Diante do exposto, o objetivo desse trabalho é discorrer sobre uma investigação matemática referente a Somas Numéricas Sequenciais, com vistas a tecer uma proposta didática investigativa, voltada para a Educação Básica, a ser analisada e implementada por licenciandos em Matemática, como parte das tarefas desenvolvidas nas disciplinas de Estágio Supervisionado de Matemática na Educação Básica, dos cursos de Licenciatura em Matemática da Universidade Federal de São Carlos (UFSCar).

Cabe destacar que a proposta didática foi elaborada a partir de duas questões diretrizes: 1 em que nível de escolaridade essa investigação matemática referente a Somas Numéricas Sequenciais poderia ser desenvolvida?; 2 - a partir de qual enfoque metodológico isso poderia ser feito em sala de aula?

\section{Fundamentação teórica}

MARTIN, W.; ZAMPIERI, M. T. Um estudo sobre somas sequenciais como propostas didáticas investigativas para o ensino de Matemática. C.Q.D.- Revista 
Pensando nessas questões enunciadas anteriormente, foi realizada uma consulta à Base Nacional Comum Curricular (BNCC), buscando procurar orientações sobre os conhecimentos e habilidades referentes ao campo da Álgebra. E então deparamo-nos com a seguinte orientação, para os anos finais do Ensino Fundamental, em que é recomendado aos alunos estabelecer "uma generalização de uma propriedade, investigar a regularidade de uma sequência numérica [...]" (BRASIL, 2018, p.267). Mais especificamente, para o $8^{\circ}$ ano, há a recomendação para o estudo de sequências recursivas e não-recursivas. E as habilidades desejáveis a serem desenvolvidas são as seguintes:

Identificar a regularidade de uma sequência numérica ou figural não recursiva e construir um algoritmo por meio de um fluxograma que permita indicar os números ou as figuras seguintes. Identificar a regularidade de uma sequência numérica recursiva e construir um algoritmo por meio de um fluxograma que permita indicar os números seguintes (BRASIL, 2018, p. 309).

Assim, podemos notar que compreender e saber expressar a regularidade de uma sequência é algo recomendado para o $8^{\circ}$ ano, de acordo com a BNCC. No currículo paulista, a recomendação é análoga, também para esse mesmo nível de escolaridade.

Contudo, sempre é importante irmos além das recomendações dos documentos oficiais, ou como bem enfatiza o pesquisador Ole Skovsmose, é importante que, enquanto professores e pesquisadores sejamos consumidores críticos desses documentos. Tanto a elaboração quanto a implementação da BNCC causaram controvérsias na comunidade acadêmica, por diferentes motivos, mas um deles foi a falta de referências sobre metodologias de ensino que já vem sendo estudadas há anos pela comunidade de Educação Matemática, especificamente. E isso remetenos a sempre pensarmos em propostas didáticas com embasamento na literatura científica e não somente em tais documentos.

Diante dessas colocações, foi elaborada uma proposta didática flexível, para que, posteriormente, pudesse ser adaptada, dependendo do contexto em que os (futuros) professores atuam. Ou seja, essa proposta foi pensada para ser debatida com estudantes do curso de Licenciatura em Matemática, com o intuito de a aprimorarmos, para que possam ser aplicadas por eles em suas atuações como estagiários na Educação Básica, uma vez que elaborar, planejar, implementar e avaliar planos de aula fazem parte da ementa das disciplinas de Estágio Supervisionado de Matemática da Educação Básica (UNIVERSIDADE FEDERAL DE SÃ̃O CARLOS, 2017). Além disso, essa proposta didática poderá ser debatida com os professores de Matemática que atuam no colégio em que o primeiro autor é proprietário, com vistas a discutir possibilidades para abordá-la com os alunos do $8^{\circ}$ ano matriculados no colégio.

Sendo assim, buscamos inspiração nas noções de ambientes de aprendizagem e cenários para investigação de Skovsmose (2000). Particularmente, no caso dessa investigação matemática, que detalharemos na seção seguinte, como só há referências à própria Matemática, pensamos em elaborar uma proposta didática em consonância com o segundo ambiente de investigação, abordado por Skovsmose (2000).

Cabe destacar que esse autor discorre sobre seis ambientes de aprendizagem, em que há três tipos de referências (Matemática Pura, semi-realidade, realidade) e dois diferentes paradigmas de práticas em sala de aula (paradigma do exercício e cenários para investigação), conforme mostra a Figura 1, a seguir: 


\begin{tabular}{|l|c|c|}
\hline & Exercícios & Cenário para Investigação \\
\hline Referências à matemática pura & $(1)$ & $(2)$ \\
\hline Referências à semi-realidade & $(3)$ & $(4)$ \\
\hline Referências à realidade & $(5)$ & $(6)$ \\
\hline
\end{tabular}

Figura 1 - Ambientes de aprendizagem Fonte: Skovsmose (2000, p. 8)

Os ambientes do tipo um são aqueles semelhantes a "exercícios de fixação", que só tem como base a Matemática Pura e que são caracterizados por apresentarem apenas uma resposta correta. Já o ambiente dois, embora também só faça referências à Matemática Pura, apresenta um enfoque investigativo. O autor apresenta então o seguinte exemplo:

Consideremos o seguinte quadro numérico apresentado a seguir:

\begin{tabular}{|c|c|c|c|c|c|c|c|c|c|}
\hline 1 & 2 & 3 & 4 & 5 & 6 & 7 & 8 & 9 & 10 \\
\hline 11 & 12 & 13 & 14 & 15 & 16 & 17 & 18 & 19 & 20 \\
\hline 21 & 22 & 23 & 24 & 25 & 26 & 27 & 28 & 29 & 30 \\
\hline 31 & 32 & 33 & 34 & 35 & 36 & 37 & 38 & 39 & 40 \\
\hline 41 & 42 & 43 & 44 & 45 & 46 & 47 & 48 & 49 & 50 \\
\hline 51 & 52 & 53 & 54 & 55 & 56 & 57 & 58 & 59 & 60 \\
\hline 61 & 62 & 63 & $\ldots$ & & & & & & \\
\hline
\end{tabular}

Quadro 1 - Uma interessante e antiga tabela de números Fonte: SKOVSMOSE (2000, p. 3)

Assim, a partir desse quadro, o autor propõe diferentes perguntas que poderiam ser feitas para os estudantes a fim de convidá-los para um processo investigativo, a partir de algumas propriedades referentes aos retângulos destacados no quadro. Por exemplo, se considerarmos os cantos do retângulos como sendo $a, b, c$ e $d$, é possível calcular $a c-b d$, e constatar, por meio de um processo investigativo, que essa propriedade é mantida mesmo quando o retângulo for transladado. Depois ele vai avançando na investigação, propondo um questionamento sobre o que acontece se girarmos em $90^{\circ}$ o retângulo, e assim por diante, ou seja, sempre avançando com perguntas do tipo: "O que acontece se....?" (SKOVSMOSE, 2000). Então esse seria o exemplo de um ambiente de aprendizagem do tipo dois.

No ambiente de aprendizagem do tipo três, um possível exemplo seria uma proposta com referência na semi-realidade, mas ainda dentro do paradigma do exercício, como aqueles problemas clássicos de livros didáticos, algo que podemos hipoteticamente exemplificar como: "Joãozinho foi na feira e comprou 5 melões a 20 reais cada. Quanto Joãozinho gastou no total? 
"Nesse ambiente, a ideia seria apenas resolver o exercício, nem ao menos teria espaço para questionar o porquê de Joãozinho comprar 5 melões e tampouco se é justo esse preço.

Já no ambiente quatro, embora também se faça uso da semi-realidade, o problema deve ser mais aberto e fomentar uma investigação. Já no ambiente de aprendizagem cinco, que tem referência na realidade, mas ainda dentro do paradigma do exercício, poderíamos exemplificar com exercícios de vestibular que apresentam algum gráfico ou alguma situação da realidade, mas que também não abrem espaço para uma investigação, ou seja, iriam apenas requerer uma resposta correta, como é característico dentro do paradigma do exercício.

Já no ambiente seis, há referências à realidade, e é um ambiente guiado pelas investigações. Skovsmose (2000) cita como exemplo o trabalho com projetos. Por exemplo, o referido autor cita como exemplo um projeto que tem em seu cerne a seguinte discussão: "A agricultura dinamarquesa está fazendo coisas erradas do ponto de vista da energia? "(SKOVSMOSE, 2000, p. 12). E então os estudantes trabalham com dados reais, de uma fazenda real, e assim, percebem que somente uma resposta correta não faz mais sentido. E assim, algumas características são marcantes desse processo investigativo:

O professor tem o papel de orientar. Novas discussões baseadas em investigação sempre surgem: como calcular a área frontal de um ciclista? O problema agora é a montagem de modelos para os cálculos de input-output, o que torna importante reflectir sobre os resultados dos cálculos. Eles são confiáveis? Considerámos os factores relevantes? Bem, podemos comparar com as estatísticas oficiais. Mas qual desses resultados é o correcto? A reflexão crítica sobre matemática e modelação matemática ganha um novo significado (SKOVMOSE; 2000, p. 13).

Assim, segundo esse autor, ao transitarmos por esses ambientes, saindo do paradigma do exercício e caminhando no sentido dos cenários para investigação, pode haver uma descentralização do papel do professor, levando os estudantes a agirem com mais autonomia. Em consonância com isso, pensamos que a investigação matemática poderia ser abordada com os estudantes de acordo com o segundo ambiente de aprendizagem, que apesar de fazer somente referência à matemática pura, apresenta um enfoque investigativo. Detalhes sobre essa investigação matemática, como sobre procedimentos metodológicos referentes a esse artigo e sobre a elaboração da proposta didática serão apresentados a seguir.

\section{Somas numéricas sequenciais: contextualização da investigação matemática realizada e metodologia de pesquisa}

Esse artigo, conforme enunciado em seu objetivo, retrata um estudo que busca discorrer sobre uma investigação matemática referente a Somas Numéricas Sequenciais, com vistas a tecer uma proposta didática investigativa, voltada para a Educação Básica, a ser analisada e implementada por licenciandos em Matemática. Desse modo, para o desenvolvimento do estudo optamos por seguir os pressupostos da metodologia de pesquisa qualitativa.

Nesse sentido, segundo Godoy (2005), é necessário que haja uma explicação cuidadosa da metodologia, de modo a descrever minuciosamente a forma como a pesquisa foi realizada. Diante disso, é importante que haja uma contextualização e uma explicitação detalhada dos procedimentos metodológicos do estudo. Contudo, embora o presente artigo tenha sido fruto de uma parceria, houve etapas que foram desenvolvidas separadamente por cada autor, e, em consonância com Godoy (2005), julgamos importante descrevê-las, com o intuito de detalhar minuciosamente como a pesquisa foi realizada e sermos coerentes com nossa opção metodológica. Assim, nos próximos parágrafos será descrita a trajetória do estudo, desde seu início. 
No final do ano de 2019, o primeiro autor desse artigo começou a refletir sobre somas numéricas sequenciais e a buscar por alguns padrões. Tudo se iniciou como uma tarefa que mantivesse sua mente ativa, mas depois de algum tempo, houve um envolvimento cada vez mais intenso com os padrões que tais sequências apresentavam, e, aproveitando o tempo de pandemia, fez um convite para a segunda autora, para que ela colaborasse, de alguma forma, para divulgar as nuances referentes a esse processo investigativo, e alguns resultados, no meio acadêmico.

Sendo assim, a segunda autora passou a estudar com mais profundidade a investigação realizada, a fim de elaborar uma proposta didática a partir dela, com vistas a ser debatida com licenciandos em Matemática, dos cursos de Licenciatura em Matemática da UFSCar, mais especificamente, nas disciplinas de Estágio Supervisionado. O intuito disso seria que os licenciados refletissem sobre a proposta e a adaptassem para poderem aplicar em suas aulas de regência na Educação Básica.

Sendo assim, o estudo contempla a realização da investigação matemática com somas numéricas sequenciais e a elaboração da proposta didática a partir dessa investigação, sendo assim de natureza qualitativa, e também de cunho documental, uma vez que fizemos uso de fontes primárias como: manuscrito com a investigação matemática realizada, documentos curriculares oficiais nacionais e estaduais (Estado de São Paulo), projeto político pedagógico do curso de Licenciatura em Matemática (UNIVERSIDADE FEDERAL DE SÃO CARLOS, 2017) da Universidade Federal de São Carlos (UFSCar) e textos teóricos. Mais precisamente, o percurso metodológico foi composto pelos seguintes momentos:

$1^{\circ}$ ) O desenvolvimento da investigação matemática (trabalho realizado pelo primeiro autor);

$2^{\circ}$ ) A análise acerca dessa investigação e adequação da temática à área de Educação Matemática. Esse momento consistiu de um estudo teórico dos manuscritos do primeiro autor, sobre os conceitos matemáticos tratados na referida investigação, buscando aproximá-la de uma abordagem que estivesse em consonância com a concepção de cenários para investigação de Skovsmose (2000). O estudo remeteu, necessariamente, a uma pesquisa bibliográfica que envolveu tanto investigações sobre o que é preconizado na Base Nacional Comum Curricular (BNCC), quanto nas diretrizes curriculares para a Educação Básica do Estado de São Paulo (SÃO PAULO, 2019). Além disso, foi realizado um estudo nas diretrizes referentes às disciplinas de Estágio Supervisionado de Matemática na Educação Básica, que constam no Projeto Político Pedagógico do Curso de Licenciatura em Matemática (UNIVERSIDADE FEDERAL DE SÃO CARLOS, 2017). Após esse momento de análise, houve a elaboração de uma proposta didática voltada para a Educação Básica, a partir dessa investigação matemática realizada, e, posteriormente, houve a escrita desse texto, dentro das normas vigentes (trabalho realizado pela segunda autora).

$3^{\circ}$ ) A revisão de todo o estudo realizado (trabalho realizado por ambos os autores).

Diante do exposto, vamos destacar primeiramente qual é a investigação matemática a que estamos nos referindo:

Inicialmente, o primeiro autor começou observando a primeira soma dos números naturais de 1 a 100, conforme já feito por Gauss (1777-1855) (GOMES, 2009), cuja resposta é 5050. E então, ele fez as seguintes indagações: Mas o que acontece se eu fizer uma segunda soma? E uma terceira soma? E uma quarta soma? E se eu gerar sequências de somas sucessivamente? Há padrões nessas sequências? Se sim, quais? Se eu agrupar cada uma dessas sequências em colunas, gerando uma tabela, haveria algum modo de descobrir um número que ocupasse uma posição específica? Foi perseguindo essas questões que o primeiro autor sintetizou suas ideias. 


\subsection{Somas Numéricas Sequenciais}

As colunas abaixo representadas são somas sequenciais ${ }^{1}$, sendo os primeiros elementos de cada coluna sempre representados pelo número 1. Na primeira coluna temos os números naturais:

\begin{tabular}{|l|l|l|l|}
\hline \multicolumn{1}{|c|}{$\mathbb{N}$} & \multicolumn{1}{|c|}{$2 \mathrm{~S}$} & \multicolumn{1}{c|}{$3 \mathrm{~S}$} \\
\hline 1 & 1 & 1 & 1 \\
\hline 2 & 3 & 4 & 5 \\
\hline 3 & 6 & 10 & 15 \\
\hline 4 & 10 & 20 & 35 \\
\hline 5 & 15 & 35 & 70 \\
\hline 6 & 21 & 56 & 126 \\
\hline 7 & 28 & 84 & 210 \\
\hline 8 & 36 & 120 & 330 \\
\hline 9 & 45 & 165 & 495 \\
\hline 10 & 55 & 220 & 715 \\
\hline$\cdot$ &. &. &. \\
. & $\cdot$ &. &. \\
\hline
\end{tabular}

Quadro 2 - Somas numéricas sequenciais, representadas até à quarta coluna.

Fonte: manuscrito do primeiro autor.

A sequência formada pelos números naturais constitui uma progressão aritmética de razão 1. A soma dos primeiros dez termos dessa sequência é representada por:

$$
\mathrm{S}_{10}=\frac{n(n+1)}{2}=55
$$

A segunda soma (2S), se os números forem apresentados de forma conveniente, eles se mostrarão com particularidades deveras interessantes. Como podemos constatar:

\begin{tabular}{|c|c|c|c|c|c|c|}
\hline 1 & & & & & & \\
\hline 2 & 2 & & & & & \\
\hline 3 & 4 & 3 & & & & \\
\hline 4 & 6 & 6 & 4 & & & \\
\hline 5 & 8 & 9 & 8 & 5 & & \\
\hline 6 & 10 & 12 & 12 & 10 & 6 & \\
\hline 7 & 12 & 15 & 16 & 15 & 12 & 7 \\
\hline
\end{tabular}

${ }^{1} 1 \mathrm{~S}$ é referente à primeira soma, $2 \mathrm{~S}$ é referente à segunda soma, e assim sucessivamente. $\mathrm{O}$ quadro pode ser expandido o quanto se queira, tanto verticalmente (para baixo) quanto horizontalmente (para o lado direito).

MARTIN, W.; ZAMPIERI, M. T. Um estudo sobre somas sequenciais como propostas didáticas investigativas para o ensino de Matemática. C.Q.D.- Revista Eletrônica Paulista de Matemática, Bauru, v. 21, p. 1-17, dez. 2021.

DOI: $10.21167 /$ cqdvol21202123169664wmmtz0117 Disponível em: http://www.fc.unesp.br/departamentos/matematica/revista-cqd/ 


\begin{tabular}{|c|c|c|c|c|c|c|c|c|c|}
\hline 8 & 14 & 18 & 20 & 20 & 18 & 14 & 8 & & \\
\hline 9 & 16 & 21 & 24 & 25 & 24 & 21 & 16 & 9 & \\
\hline 10 & 18 & 24 & 28 & 30 & 30 & 28 & 24 & 18 & 10 \\
\hline$\cdot$ & . & $\cdot$ & $\cdot$ & $\cdot$ & • & . & $\begin{array}{l}\cdot \\
\cdot \\
.\end{array}$ & $\cdot$ & . \\
\hline
\end{tabular}

Figura 2 - Triângulo numérico $(2 \mathrm{~S})$

Observações pertinentes:

Fonte: manuscrito do primeiro autor.

1 - Todas as linhas são simétricas².

2 - As linhas decrescem do centro para as extremidades.

3 - As linhas pares apresentam dois elementos centrais pares.

4 - As linhas ímpares apresentam apenas um elemento central.

5 - As linhas pares decrescem do centro para as extremidades em números pares crescentes sequenciais.

6 - As linhas ímpares o fazem em números ímpares crescentes e sequenciais.

7 - O elemento central da linha par, que aparece duas vezes, pode ser determinado por:

$$
\mathrm{E}_{\mathrm{c}} \mathrm{p}=\frac{l}{2} \cdot\left(\frac{l+2}{2}\right)
$$

Em que $\mathrm{E}_{\mathrm{c}}^{\mathrm{p}}$ é o elemento central da linha par e $l$ representa a linha em que ele se encontra. 8 - O elemento central da linha ímpar pode ser determinado por:

$$
E_{c}{ }^{i}=\left(\frac{l+1}{2}\right)^{2}
$$

Em que $E_{c}{ }^{i}$ é elemento central da linha ímpar e $l$ representa a linha em que ele se encontra. 9 - Qualquer elemento ${ }^{3}$ pode ser determinado por:

$$
\mathrm{E}_{\mathrm{q}}=c(l-c+1)
$$

Em que $\mathrm{E}_{\mathrm{q}}$ é o elemento qualquer e $l$ e $c$ representam a linha e a coluna em que ele se encontra, respectivamente.

10 - A soma de todos os elementos de uma linha corresponde a soma $(2 \mathrm{~S})$ até o número desejado.

11 - A soma da soma (2S) pode ser obtida de modo mais racional. Como exemplo, será utilizada a linha 10 , ou seja, $l=10$

Procedimento para obter a soma da soma, até uma determinada linha que se queira:

${ }^{2}$ Ou seja, os elementos que aparecem em uma mesma linha estão em posições simétricas.

${ }^{3}$ Qualquer elemento cujo índice da coluna seja menor que o índice da linha, ou seja, em que c $<1$.

MARTIN, W.; ZAMPIERI, M. T. Um estudo sobre somas sequenciais como propostas didáticas investigativas para o ensino de Matemática. C.Q.D.- Revista Eletrônica Paulista de Matemática, Bauru, v. 21, p. 1-17, dez. 2021.

DOI: $10.21167 /$ cqdvol21202123169664wmmtz0117 Disponível em: http://www.fc.unesp.br/departamentos/matematica/revista-cqd/ 
Parte-se do pressuposto que todos os elementos dessa linha tenham o mesmo valor do elemento central:

Como o elemento central $\left(\mathrm{E}_{\mathrm{c}}\right)$ é igual a 30 , temos o seguinte:

$$
L 10 \Rightarrow E c=30 \quad S 10=10 \times 30=300
$$

Vejamos agora o que devemos subtrair desse valor para que se possa obter o valor real da linha 10. Em conformidade com a quinta observação:

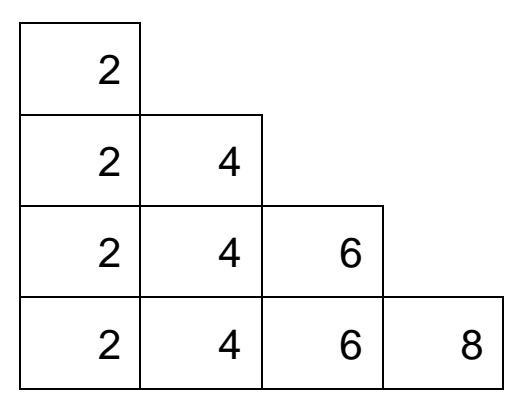

Ou, se somarmos os elementos de cada linha, teremos:

2

6

12

20

Cuja soma resulta em 40 .

Por simetria: $40 \times 2=80$

Então o valor real de S10 será:

$\mathrm{S} 10=300-80=220$

Ou ainda, podemos pensar em duas representações quadradas ao invés da triangular mostrada anteriormente, utilizando apenas o número 2. Basta transformar cada casa que contém o número 4 em duas casas com o número 2, cada casa que contém o número $6 \mathrm{em}$ três casas que contém o número 2 , e, cada casa que contém o número 8 em quatro casas que contém o número 2 , resultando em duas representações quadradas, sendo uma com quatro linhas por quatro colunas $(4 \times 4)$ e a outra com duas linhas por duas colunas $(2 \times 2)$, conforme pode-se observar a seguir:

\begin{tabular}{|l|l|l|l|}
\hline 2 & 2 & 2 & 2 \\
\hline 2 & 2 & 2 & 2 \\
\hline 2 & 2 & 2 & 2 \\
\hline 2 & 2 & 2 & 2 \\
\hline
\end{tabular}

Quadrado de quatro linhas por quatro colunas $(4 \mathrm{x} 4)$ 


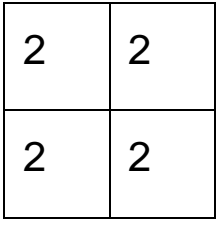

Quadrado de duas linhas por duas colunas (2x2)

Levando em consideração a simetria, o seguinte cálculo é efetuado:

$2[2(4 \times 4)+2(2 \times 2)]=80$

Então o valor real de S10 será:

$\mathrm{S} 10=300-80=220$

De modo análogo, podemos proceder para se obter soma de ímpares (de acordo com a observação número 6). Vamos obter o valor real da soma da nona linha:

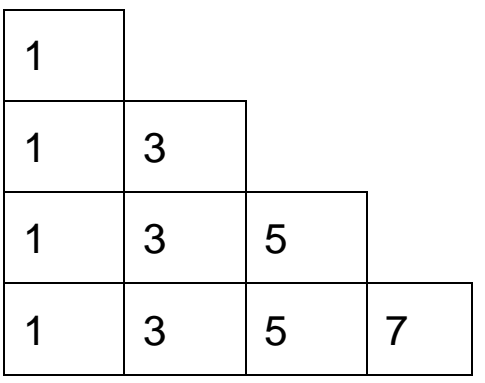

Ou, se somarmos os elementos de cada linha, teremos:

1

4

9

16

$\mathrm{Ou}$

$1^{2}$

$2^{2}$

$3^{2}$

$4^{2}$

Cuja soma resulta em 30

Por simetria, temos que $2 \times 30=60$

S9 $=9 \times 25-60=165$, ou seja

$\mathrm{S} 9=165$

\section{Observações úteis}

Para linhas pares: é possível determinar os valores a serem subtraídos do valor pressuposto:

Exemplo: linha 12, ou seja, $l=12$

$m=(l-2) / 2=(12-2) / 2=5$ (maior par), ou seja, devemos subtrair até o quinto maior par, que é 10 . Vamos verificar:

Consideremos a linha 12 , representada a seguir:

\begin{tabular}{|l|l|l|l|l|l|l|l|l|l|l|l|}
\hline 12 & 22 & 30 & 36 & 40 & 42 & 42 & 40 & 36 & 30 & 22 & 12 \\
\hline
\end{tabular}


O elemento central é o 42, procedendo de forma análoga aos exemplos anteriores, primeiro supomos que a soma desses elementos é 42 x $12=504$.

Precisamos subtrair os elementos, de acordo com a representação triangular a seguir, tendo o 10 como o maior par representado:

\begin{tabular}{|c|c|c|c|c|}
\hline 2 & & & & \\
\hline 2 & 4 & & & \\
\hline 2 & 4 & 6 & & \\
\hline 2 & 4 & 6 & 8 & \\
\hline 2 & 4 & 6 & 8 & 10 \\
\hline
\end{tabular}

Então, se somarmos os elementos de cada linha, teremos:

$$
\begin{aligned}
& 2 \\
& 6 \\
& 12 \\
& 20 \\
& 30
\end{aligned}
$$

Cuja soma resulta em 70 .

Por simetria: 70 × $2=140$

Então o valor real de S12 será:

$\mathrm{S} 12=504-140=364$

Exemplo: $l=14$

$m=(l-2) / 2=(14-2) / 2=6$ (maior par), ou seja, devemos subtrair até o sexto maior par, que é 12 .

E para verificar, podemos prosseguir de modo análogo ao exemplo anterior.

Para linhas ímpares, a regra é mais simples, conforme a seguir:

Exemplo: $l=11$

$$
I=l-2 \text {. }
$$

$\mathrm{I}=11-2=9$ (maior ímpar a ser considerado na representação triangular), conforme representação a seguir:

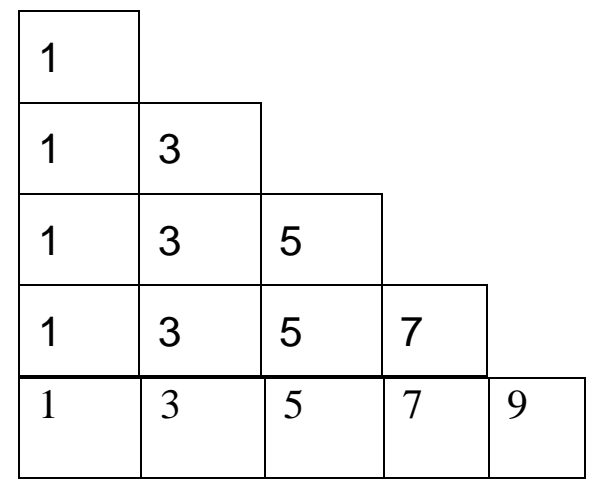




\subsection{Soma da soma da soma (3S)}

Tomando-se como base o triângulo numérico 2S (Figura 1), podemos somar cada coluna, e, posteriormente, somar essas somas, assim obteremos $3 \mathrm{~S}$, considerando a soma dos elementos até a décima linha:

$55+90+108+112+105+90+70+48+27+10$

Total $=715$

Então surge uma pergunta:

Há, sem uso de matemáticas mais sofisticadas, a possibilidade de obter-se números "grandes o suficiente" sem ter que somar exaustivamente cada um deles?

Para responder essa pergunta, o quadro a seguir foi construído:

\begin{tabular}{|l|l|l|l|l|l|l|l|l|l|l|l|}
\hline 1 & 1 & 1 & 1 & 1 & 1 & 1 & 1 & 1 & 1 & 1 & 1 \\
\hline $\mathbf{2}$ & 3 & 4 & 5 & 6 & 7 & 8 & 9 & 10 & 11 & 12 & 13 \\
\hline 3 & $\mathbf{6}$ & 10 & 15 & 21 & 28 & 36 & 45 & 55 & 66 & 78 & 91 \\
\hline 4 & 10 & $\mathbf{2 0}$ & 35 & 56 & 84 & 120 & 165 & 220 & 286 & 364 & 455 \\
\hline 5 & 15 & 35 & $\mathbf{7 0}$ & 126 & 210 & 330 & 495 & 715 & 1001 & 1365 & 1820 \\
\hline 6 & 21 & 56 & 126 & $\mathbf{2 5 2}$ & 462 & 792 & 1287 & 2002 & 3003 & 4368 & 6188 \\
\hline 7 & 28 & 84 & 210 & 462 & $\mathbf{9 2 4}$ & 1716 & 3003 & 5005 & 8008 & 12376 & 18564 \\
\hline 8 & 36 & 120 & 330 & 792 & 1716 & $\mathbf{3 4 3 2}$ & 6435 & 11440 & 19448 & 31824 & 50388 \\
\hline 9 & 45 & 165 & 495 & 1287 & 3003 & 6435 & $\mathbf{1 2 8 7 0}$ & 24310 & 43758 & 75582 & 125970 \\
\hline 10 & 55 & 220 & 715 & 2002 & 5005 & 11440 & 24310 & $\mathbf{4 8 6 2 0}$ & 92378 & 167960 & 293930 \\
\hline 11 & 66 & 286 & 1001 & 3003 & 8008 & 19448 & 43758 & 92378 & $\mathbf{1 8 4 7 5 6}$ & 352716 & 646646 \\
\hline 12 & 78 & 364 & 1365 & 4368 & 12376 & 31824 & 75582 & 167960 & 352716 & $\mathbf{7 0 5 4 3 2}$ & 1352678 \\
\hline 13 & 91 & 455 & 1820 & 6188 & 18564 & 50388 & 125970 & 293930 & 646646 & 1352078 & $\mathbf{2 7 0 4 1 5 6}$ \\
\hline
\end{tabular}

Quadro 3 - Quadro numérico com somas sequenciais até $14^{\mathrm{a}}$ linha e $12^{\mathrm{a}}$ coluna.

Fonte: manuscrito do primeiro autor.

Observando-se o quadro, nota-se que há um eixo (seria de simetria se desconsiderássemos a primeira linha composta somente pelo número 1), o qual destacamos em negrito. Os elementos desse eixo são da forma $\mathrm{e}_{\mathrm{ij}} \operatorname{com} i=j+1$, sendo $i$ o índice referente à linha ocupada pelo referido elemento e $j$ a coluna. Observemos que esses elementos são divisíveis por $i$, vejamos:

$\mathrm{e}_{32}=6.6$ é divisível por 3 , cujo resultado é 2 .

$\mathrm{e}_{43}=20.20$ é divisível por 4 , cujo resultado é 5 .

$\mathrm{e}_{54}=70.70$ é divisível por 5, cujo resultado é 14 .

$\mathrm{e}_{65}=252.252$ é divisível por 6 , cujo resultado é 42 .

$\mathrm{e}_{76}=924.924$ é divisível por 7, cujo resultado é 132 . 
$\mathrm{e}_{87}=3432.3432$ é divisível por 8 , cujo resultado é 429 .

$\mathrm{e}_{98}=12870.12870$ é divisível por 9, cujo resultado é 1430 .

$\mathrm{e}_{109}=48620.48620$ é divisível por 10, cujo resultado é 4862 .

$\mathrm{e}_{1110}=184756.184756$ é divisível por 11 , cujo resultado é 16796 .

$\mathrm{e}_{1211}=705432.705432$ é divisível por 12, cujo resultado é 58786 .

$\mathrm{e}_{1312}=2704156,2704156$ é divisível por 13, cujo resultado é 208012.

Com os quocientes obtidos podemos obter outros elementos. Em conformidade:

1) $2 \times e_{98}-1430=24310$

$24310=\mathrm{e}_{99}=\mathrm{e}_{108}$

2) $1 \times \mathrm{e}_{98}-1430=24310-1430=11440$

$11440=\mathrm{e}_{107}=\mathrm{e}_{89}$

3) $\frac{1}{2} \times e_{98}-1430=24310-1430=5005$

$11440=\mathrm{e}_{79}=\mathrm{e}_{106}$

4) $\mathrm{e}_{87}-1430=3432-1430=2002$

$2002=\mathrm{e}_{69}=\mathrm{e}_{105}$

Podemos obter outros elementos como $\mathrm{e}_{59}, \mathrm{e}_{49}$ por outro processo.

a) Obtenção de $\mathrm{e}_{59}$

$$
\begin{aligned}
& \mathrm{e}_{59}=\mathrm{e}_{79}-\left(\mathrm{e}_{78}+\mathrm{e}_{68}\right) \\
& \mathrm{e}_{59}=5005-(3003+1287)=715 \\
& \mathrm{e}_{59}=715=\mathrm{e}_{104}
\end{aligned}
$$

b) Obtenção de $e_{49}$

$$
\begin{aligned}
& \mathrm{e}_{49}=\mathrm{e}_{69}-\left(\mathrm{e}_{68}+\mathrm{e}_{58}\right) \\
& \mathrm{e}_{49}=2002-(1287+495)=220 \\
& \mathrm{e}_{49}=220=\mathrm{e}_{103}
\end{aligned}
$$

Mas! Como podemos obter um elemento central "futuro" para se trabalhar como acima? Uma fórmula foi obtida, conforme apresentado a seguir, considerando $\mathrm{e}_{\mathrm{c}}{ }^{\mathrm{q}}$ como sendo um elemento central qualquer:

$\mathrm{e}_{\mathrm{c}}{ }^{\mathrm{q}}=\frac{2^{n-2} \cdot \prod_{5}^{2 n-3} x}{\prod_{4}^{n-1} k}$, em que $x \in \mathbb{N}$ ímpares, $k \in \mathbb{N}$.

E $n \in \mathbb{N}$, e representa o índice da linha em que se encontra esse elemento.

Por exemplo, vamos encontrar o elemento central $\mathrm{e}_{1110}$ :

$\mathrm{e}_{1110}=\frac{2^{11-2} \cdot \prod_{5}^{2 \cdot 11-3} x}{\prod_{4}^{11-1} k}$, em que $\mathrm{x} \in \mathbb{N}$ ímpares, $\mathrm{k} \in \mathbb{N}$.

$\mathrm{e}_{1110}=\frac{2^{9} \cdot 5 \cdot 7 \cdot 9 \cdot 11 \cdot 13 \cdot 15 \cdot 17 \cdot 19}{4 \cdot 5 \cdot 6 \cdot 7 \cdot 8 \cdot 9 \cdot 10}=2^{2} \cdot 11 \cdot 13 \cdot 17 \cdot 19=184756$

$\mathrm{e}_{1110}=184756$ 
Tendo-se um elemento central "futuro", como obtivemos acima, podemos obter os demais, de forma menos "trabalhosa". Partindo-se do elemento obtido, $\mathrm{e}_{1110}$, podemos obter o elemento $\mathrm{e}_{1211}$.

$$
\begin{aligned}
& \mathrm{e}_{1211}=\frac{\mathrm{ec} 11 \mathrm{x} 10}{11} \cdot 2 \cdot(11+10) \\
& \mathrm{e}_{1211}=16796 \mathrm{x} 42=705432
\end{aligned}
$$

Descrição da fórmula:

As referências serão sempre o quadro numérico (Quadro 2). Quando fizermos referência a alguma linha ou coluna, as unidades serão desconsideradas.

As investigações começaram a partir do elemento central 20, que está na quarta linha, ou seja o e 43 .

1 - O número 20 também pode ser escrito por $10+10$ ou 4.5. Cinco é a soma de 2 com 3 (que são os índices das duas linhas anteriores à linha 4), que se multiplicado por 2 dará 10. Se, novamente, multiplicar por 2 , teremos $20,10+10$, conforme representado a seguir:

2.2. $(2+3)=20$

2 - O mesmo procedimento será repetido para o número 70:

2.2.2. $(2+3) \cdot(3+4)=280$

Se dividirmos por 4 obteremos 70 .

3 - Idem para 252:

2.2.2.2. $(2+3) \cdot(3+4) \cdot(4+5)=5040$

Esse valor assim obtido dividido por $20=4.5$, obteremos 252 .

4 - O mesmo vale para 924 :

2.2.2.2.2.(2+3). $(3+4) .(4+5) \cdot(5+6)=110880$

Se dividirmos por 120 , obteremos 924 . O valor 120 é, na verdade, 4.5.6.

Com base nesses resultados, houve uma generalização:

1 - Os índices das linhas, como foram somados, sempre se obterá números ímpares acima de 5 .

2 - A quantidade de " 2 " sempre será uma vez a mais que a quantidade das somas das duplas de ímpares entre parênteses.

3 - No denominador teremos um produto de números naturais a partir de 4 .

A conclusão é a fórmula:

$\mathrm{e}_{\mathrm{c}}{ }^{\mathrm{q}}=\frac{2^{n-2} \cdot \prod_{5}^{2 n-3} x}{\prod_{4}^{n-1} k}$, em que $x \in \mathbb{N}$ ímpares, $k \in \mathbb{N}$.

E $n \in \mathbb{N}$, e representa o índice da linha em que se encontra esse elemento.

\section{Pensando em um cenário para investigação em sala de aula}

A partir dessa investigação matemática descrita anteriormente, pensamos em uma proposta didática $^{4}$ que articularia momentos de trabalho individual com trabalhos em grupo, de forma

${ }^{4}$ É uma proposta ainda sem definição de carga horária e ainda com lacunas a serem preenchidas, pois a ideia é que os licenciandos e os professores também tragam suas contribuições para o seu aprofundamento, pensando depois em elaborar planos de aula com base nisso, levando em consideração seus contextos de atuação e suas preferências metodológicas.

MARTIN, W.; ZAMPIERI, M. T. Um estudo sobre somas sequenciais como propostas didáticas investigativas para o ensino de Matemática. C.Q.D.- Revista Eletrônica Paulista de Matemática, Bauru, v. 21, p. 1-17, dez. 2021.

DOI: $10.21167 /$ cqdvol21202123169664wmmtz0117 Disponível em: http://www.fc.unesp.br/departamentos/matematica/revista-cqd/ 
que os estudantes fariam registros escritos relatando todo o processo de desenvolvimento das tarefas, e, arquivariam em seus portfólios, sendo esses um dos elementos avaliativos, junto à observação contínua do desenvolvimento das tarefas em sala de aula ${ }^{5}$.

Sendo assim, em um primeiro momento, os estudantes trabalhariam individualmente, e seriam convidados a efetuar as somas, conforme o primeiro autor começou a fazer, indo até a terceira soma, sendo instigados a refletirem sobre as seguintes questões: Mas o que acontece se eu fizer uma segunda soma? E uma terceira soma? E uma quarta soma? E se eu gerar sequências de somas sucessivamente? Há padrões nessas sequências? Se sim, quais? Se eu agrupar cada uma dessas sequências em colunas, gerando uma tabela, haveria algum modo de descobrir um número que ocupasse uma posição específica?

Em seguida, ainda individualmente, convidaríamos os alunos para pensarem sobre a representação triangular da segunda soma, conforme mostrado na Figura 2, com questões que os instigassem a elencarem as propriedades que observam em relação a essa sequência.

Em outro momento, os estudantes seriam convidados a trabalharem em pequenos grupos, no máximo com três membros em cada, para confrontarem suas respostas, debatendo as ideias e fazendo pequenos ajustes, se considerarem necessário. É importante ressaltar que o professor seria orientador ao longo de todo esse processo, tirando as dúvidas dos alunos sempre que preciso.

Dando prosseguimento ao desenvolvimento da proposta, uma segunda etapa ocorreria no laboratório de informática e poderia ser dividida em três momentos: o primeiro seria a realização de uma dinâmica de aula no laboratório de informática, com o software Excel da Microsoft Office ou com alguma outra planilha de cálculo, em que os estudantes, em duplas ou trios, seriam convidados a pensar em uma forma de automatizar o preenchimento do quadro numérico apresentado anteriormente (Quadro 3), com o intuito de desenvolver uma habilidade preconizada na BNCC e nas Diretrizes Curriculares Tecnologia e Inovação, referente à tradução de uma determinada situação-problema para outras linguagens, tais como algoritmos, fórmulas, fluxogramas, etc. (BRASIL, 2018; SÃO PAULO, 2019).

Dessa forma, inicialmente, a ideia seria que os estudantes fossem convidados a compreenderem o processo de construção de cada soma, para então construírem a tabela, ainda de forma manual, utilizando a planilha de cálculo. Depois, em um segundo momento, perguntaríamos a eles se poderia existir uma maneira de tornar esse preenchimento automático, ou pelo menos parte das células. Antes de adentrarmos às especificidades das fórmulas, perguntaríamos algo como: A célula B2 deve ser a soma dos números que estão em quais células? [Eles supostamente poderiam responder: "A1 com A2". E prosseguiríamos com as questões: E a B3? [Eles poderiam dizer: $\mathrm{A} 3$ com $\mathrm{B} 2]$. E então reforçaríamos que, como já teríamos esses valores, poderíamos calcular o valor referente a B3 em função desses que já existem, e assim prosseguiríamos sucessivamente com o questionamento. Depois, a ideia seria que eles explorassem o software, realizando simulações, de modo a descobrirem como colocar essas fórmulas e como arrastá-las para as demais células, para o preenchimento automático do restante da tabela, contando com a colaboração do(a) professor(a) e dos estagiários.

O segundo momento se daria em sala de aula, cujo propósito seria que, ainda trabalhando nessas mesmas duplas ou trios, os estudantes fossem convidados, por meio de perguntas diretrizes, a fazer as investigações necessárias para concluírem as 11 observações apresentadas anteriormente, sobre a soma $2 \mathrm{~S}$, levando em consideração a investigação que já teriam feito individualmente na primeira etapa da proposta relatada anteriormente; e o terceiro momento seria, também por meio de perguntas diretrizes, que os estudantes concluíssem a fórmula a que chegou o primeiro autor, para o cálculo de um elemento central qualquer. Nesse sentido, o grande

\footnotetext{
${ }^{5}$ Pensamos em propostas didáticas a serem desenvolvidas em um contexto presencial, contudo, são passíveis de serem adaptadas para o desenvolvimento remoto.

MARTIN, W.; ZAMPIERI, M. T. Um estudo sobre somas sequenciais como propostas didáticas investigativas para o ensino de Matemática. C.Q.D.- Revista Eletrônica Paulista de Matemática, Bauru, v. 21, p. 1-17, dez. 2021.

DOI: 10.21167/cqdvol21202123169664wmmtz0117 Disponível em: http://www.fc.unesp.br/departamentos/matematica/revista-cqd/
} 
desafio nesse terceiro momento, com base nos relatos das vivências dos estagiários no ambiente escolar, será formular questões que levem os estudantes do $8^{\circ}$ ano a aceitarem o convite da investigação (SKOVSMOSE, 2000) e a constatarem os mesmos padrões observados pelo primeiro autor, além de outros que por ventura se manifestem.

Diante do exposto, gostaríamos de ressaltar que, a investigação realizada contribuiu não somente para se chegar a esses resultados matemáticos interessantes, mas também para pensarmos nessa proposta didática inicial de ensino, a ser melhor formulada e refinada em conjunto com (futuros) professores de Matemática. O grande desafio que temos pela frente é incorporarmos essa nossa aprendizagem em nossas rotinas de trabalho após esse período de pandemia. Então a pergunta que temos em mente no momento é: como fazer isso? Não temos aqui respostas prontas e acabadas, mas algo que poderemos fazer, e que ficou tão presente nesta experiência, é prestarmos mais atenção nas pessoas com as quais estamos interagindo, em especial nos estudantes, e nesse triste período, vimos a importância de deixar aflorar a criatividade, característica tão própria da espécie humana, e que nem sempre temos tempo para leva-la em consideração em nossas atividades cotidianas.

\section{Considerações finais}

Nesse artigo, tivemos o propósito de discorrer sobre uma investigação matemática referente a Somas Numéricas Sequenciais, com vistas a tecer uma proposta didática de cunho investigativo, voltada para a Educação Básica, a ser analisada e implementada por licenciandos em Matemática, como parte das tarefas desenvolvidas nas disciplinas de Estágio Supervisionado de Matemática na Educação Básica, que compõe a grade curricular do curso de Licenciatura em Matemática, da Universidade Federal de São Carlos (UFSCar).

Pensamos ainda em uma proposta inicial, flexível, para que seja debatida e aprimorada junto a professores e a futuros professores de Matemática, a partir de um processo de Imaginação Pedagógica (MILANI, 2015), que consiste em criar situações de diálogos imaginários em sala de aula, pautadas por experiências anteriores em que os envolvidos tiveram a oportunidade de ensinar, com a finalidade de compreender que possíveis imprevistos poderiam acontecer no desenvolvimento das tarefas e que soluções seriam requeridas. Embora não seja possível controlar tudo o que pode vir a acontecer, esse processo contribui para a criação de um repertório de ideias para se trabalhar com propostas investigativas.

Por fim, almejamos que as reflexões compartilhadas nesse artigo possam fomentar novas ideias para o desenvolvimento, tanto de investigações matemáticas, como de propostas didáticas que possam incentivar a autonomia dos estudantes em seus próprios processos de aprendizagem. Ademais, pretendemos instigar outros professores e futuros professores a refletirem criticamente sobre essa proposta e a adaptarem, de acordo com seus contextos de atuação.

\section{Agradecimentos}

Agradecemos ao genro de Walter Martin, Prof. Dr. Rafael Nóbrega Stipp (in memorian), que o incentivou a seguir sua intuição no trabalho com investigações matemáticas.

\section{Referências}

MARTIN, W.; ZAMPIERI, M. T. Um estudo sobre somas sequenciais como propostas didáticas investigativas para o ensino de Matemática. C.Q.D.- Revista Eletrônica Paulista de Matemática, Bauru, v. 21, p. 1-17, dez. 2021.

DOI: $10.21167 /$ cqdvol21202123169664wmmtz0117 Disponível em: http://www.fc.unesp.br/departamentos/matematica/revista-cqd/ 
BRASIL. Ministério da Educação. Base Nacional Comum Curricular - BNCC. Brasília, DF: MEC, 2018.

D’AMBROSIO, U. A educação matemática e o estado do mundo: desafios. Em Aberto, Brasília, v. 27, n. 91, p. 157-169, 2014.

GODOY, A. S. Refletindo sobre critérios de qualidade da pesquisa qualitativa. Revista Eletrônica de Gestão Organizacional, v. 3, n. 2, p. 81-89, mai./ago. 2005.

GOLDENBERG, M. A arte de pesquisar: como fazer pesquisa qualitativa em Ciências Sociais. 3. ed. Rio de Janeiro: Record, 1999.

GOMES, A. M. D. Gauss, o príncipe da matemática. [Niterói]: IM/UFF, [2009]. Disponível em: http://webeduc.mec.gov.br/portaldoprofessor/matematica/curiosidades/curiosidadesmatematicashtml/audio-gaussbr.html\#: :text=Percebeu $\% 20$ ent $\%$ C3\% A3o\%20que $\% 2 \mathrm{C} \% 20$ na $\% 20$ verdade, maiores $\% 20$ matem $\% \mathrm{C} 3 \% \mathrm{~A} 1 \mathrm{ti}-$ cos\%20que\%20j\%C3\%A1\%20existiram. Acesso em: 17 ago. 2020

MILANI, R. O processo de aprender a dialogar por futuros professores de matemática com seus alunos no estágio supervisionado. 2015. 239 p. Tese (Doutorado em Educação Matemática) - Instituto de Geociências e Ciências Exatas, Universidade Estadual Paulista "Júlio de Mesquita Filho", Rio Claro, 2015. Disponível em: http://hdl.handle.net/11449/124074. Acesso em: 22 mar. 2021.

SÃO PAULO. Diretrizes Curriculares tecnologia e inovação. [S.l.: s.n.], 2019. Disponível em: https://efape.educacao.sp.gov.br/curriculopaulista/wpcontent/uploads/sites/7/2020/02/diretrizes-curriculares-tecnologia-e-inovacao.pdf. Acesso em: 22 mar. 2021.

SKOVSMOSE, O. Cenários para investigação. Bolema, Rio Claro, v. 13, n. 14, 2000.

UNIVERSIDADE FEDERAL DE SÃO CARLOS. Curso de graduação: licenciatura em matemática: projeto pedagógico. São Carlos: UFSCar, 2017. 